\title{
The impact of a bundled intrahospital transfer protocol on the safety of critically ill patients in a South African Metropolitan Hospital System
}

\author{
L Geldenhuys, ${ }^{1}$ (D) R Wise, ${ }^{1,2}$ (D) R Rodseth ${ }^{1,3}$ iD \\ ${ }^{1}$ Discipline of Anaesthesia and Critical Care, School of Clinical Medicine, University of KwaZulu-Natal, South Africa \\ ${ }^{2}$ John Radcliffe Hospital, Intensive Care Department, Oxford University Trust Hospitals, United Kingdom \\ ${ }^{3}$ Drs Jones, Bhagwan and Partners, Pietermaritzburg, South Africa \\ Corresponding author, email: liezegeldenhuys@gmail.com
}

Background: Intrahospital transfer (IHT) of critically ill patients is associated with a high incidence of adverse events (AEs). This study aimed to determine whether the introduction of an intervention bundle could decrease AEs during, and immediately after IHT to the intensive care unit (ICU), as compared to event rates prior to the bundle's introduction.

Methods: This was a prospective, pre- and post-intervention trial, conducted in both a regional and tertiary hospital in Pietermaritzburg, South Africa. The intervention bundle consisted of an IHT protocol, a transport backpack, emergency drug container and simulation training. Primary outcomes were: 1) composite outcome of serious AEs, and 2) composite outcome of AEs contributing directly to morbidity or mortality. Secondary outcomes were miscellaneous complications, equipment-related AEs, total number of AEs, total number of IHTs complicated by AEs and the subjective measure of IHTs needing intervention within the first 30 minutes after arrival in ICU.

Results: There were 381 pre-intervention IHTs and 264 post-intervention IHTs with one documented serious AE. Adverse events directly contributing to morbidity or mortality showed a reduction from $58.3 \%$ (Cl $0.53-0.63$ ) pre-intervention, to $56.1 \%(\mathrm{Cl}$ $0.50-0.62)$ post-intervention ( $p=0.6)$. Miscellaneous complications yielded a reduction of $12.9 \%(\mathrm{Cl} 10.3-14.7 \%)$ pre-intervention to $9.5 \%(\mathrm{Cl} 8.3-11.1 \%)$ post-intervention $(p=0.2)$. Equipment-related $\mathrm{AE}$ reduced from $5.2 \%(\mathrm{Cl} 3.4-8 \%)$ to $1.9 \%(\mathrm{Cl} 0.8-4.5 \%)$ $(p=0.03)$. The total number of AEs reduced from $5 \%(\mathrm{Cl} 3.6-7.4 \%)$ to $4.1 \%(\mathrm{Cl} 2.4-6.8 \%)(p=0.03)$, while the total number of transfers complicated by AEs reduced from $63.3 \%(\mathrm{Cl} 61.9-65.1 \%)$ to $60.6 \%(\mathrm{Cl} 58.8-63.1 \%)(p=0.5)$. There was a reduction in IHTs requiring intervention within the first 30 minutes of arrival in ICU ( $34.6 \%$ to $22.7 \% ; p=0.001$ ).

Conclusion: These results support the use of an intervention bundle to decrease the incidence of AEs during IHT.

Keywords: intrahospital transfer, bundle, critical care, safety, South Africa

\section{Introduction}

Secondary transport of critically ill patients, whether inter- or intrahospital transfer (IHT), is a stressful, high-risk endeavour. ${ }^{1,2}$ Although some adverse events (AEs) are inevitable, others are preventable. These AEs directly or indirectly contribute to morbidity or mortality, by increasing the incidence of long-term complications such as pneumothoraces, atelectasis, deep vein thrombosis, prolonged length of intensive care unit (ICU) stay, ${ }^{3}$ ventilator-associated pneumonia, ${ }^{4}$ raised intracranial pressure and cardiac arrest. ${ }^{5}$

Reported AE rates during IHT vary widely and depend on the circumstances surrounding the IHTs. With a dedicated IHT team, $\mathrm{AE}$ rates as low as $1.7 \%$ have been reported, ${ }^{6}$ while other studies report rates of $22.2 \%$ and $67.9 \%$ for IHTs from the emergency department to the ICU. ${ }^{5,7}$ Serious adverse events (SAE), defined as events which would likely lead to mortality without an intervention, ${ }^{5,8}$ have been reported to be as high as $8.9-16.8 \% .5,9$ Different definitions adopted by authors make interpreting these discrepant $A E$ rates difficult. ${ }^{8}$

The aim of our study was to determine whether the introduction of an intervention bundle improved IHT quality when critically ill patients were transported into the ICU by non-ICU staff. We further aimed to determine whether the bundle decreased the incidence of AEs during and immediately after transport as compared to event rates prior to the bundle's introduction. A specific point of focus was on transfers from the operating room to ICU as there is paucity of literature regarding this group.

\section{Methods}

This study was a prospective, pre- and post-intervention trial conducted in both a regional (six-bed adult ICU) and tertiary hospital (10-bed adult ICU) in Pietermaritzburg, KwaZuluNatal, South Africa. Both ICUs were mixed medical and surgical and run as closed units. All critically ill patients undergoing IHT from the emergency department or operating theatre to ICU were included. Both new ICU admissions and ICU reinstallations (patients returning to ICU postoperatively) were included. ${ }^{10}$ Critically ill patients were defined as those requiring cardiovascular, respiratory, renal or neurological organ support, needing ICU admission for mechanical ventilation, inotropic infusions, intensive monitoring or other supportive measures. Transfers of patients performed by permanent or temporary ICU staff members to or from ICU, were excluded. A convenience sample of consecutive transfers into the ICUs was used.

Ethical approval was obtained from both hospitals' management, the University of KwaZulu-Natal's Biomedical Research Ethics Committee (BREC reference number BFC 047/17), and the 
KwaZulu-Natal Department of Health. Individual participant written consent was waived by the ethics committee, as this was a multi-institutional quality improvement study, based on systems changes that were applied to all critically ill patients undergoing $\mathrm{IHT}$.

Pre- and post-intervention data were collected on a case report form by the receiving ICU practitioner. This form included vital signs before and after transfer, AEs occurring during $\mathrm{IHT}$ and corrective steps necessary during or immediately after transfer. A transport identification code was allocated to each case report form, to protect patient confidentiality. Data was transcribed onto a spreadsheet on a password protected computer, held in a secure location. Pre-intervention data collection commenced on 1 July 2017. After the pre-intervention data collection was concluded, the intervention bundle was introduced on 27 November 2017.

The intervention bundle consisted of: 1) an IHT protocol, summarised as a checklist (Appendix 1 and 2); 2) a transport backpack and emergency drug container based on the IHT protocol's inventory and drug lists (Appendix 3); and 3) IHT simulation training which included small group practical simulation training for interns and junior medical officers, and videographic training using a simulator which was screened at meetings and teaching opportunities in the departments of anaesthesia, emergency medicine and critical care.

The protocol and checklist were based on guidelines, recommendations and checklists published in the literature, ${ }^{10-15}$ combined with expert opinion from specialist intensivists in the hospital complex. The checklist was a formal documentation of the transport, aiding in handover of the patient and became part of the patient's hospital notes. There was a subsequent induction period from 27 November 2017 to 4 January 2018, as staff involved in IHTs were informed about the bundle and the educational component was introduced. For continuity, data collection continued during the induction period, but was not included in the statistical analysis. Only the pre- and post-intervention data were compared to each other. Post- intervention data collection commenced on 5 January 2018 and concluded on 31 May 2018.

To determine the study sample size, data were used from a 2014 audit in the hospital complex which demonstrated an $\mathrm{AE}$ rate of $12.7 \%$ in patients undergoing IHT. This was likely an underestimation, as it was not the primary focus of the audit. Using an estimated pre-intervention $\mathrm{AE}$ rate of $25 \%$ and anticipated $A E$ rate of $15 \%$ after intervention, with an alpha error of 0.05 and power of 0.8 , the target sample size was calculated as 250 transfers per group. Stata 14 (StataCorp. 2015. Stata Statistical Software: Release 14. College Station, TX: StataCorp LP) was used for data analysis.

The main study outcome was to determine whether there was a significant decrease in the $A E$ rate after implementing the intervention bundle. The primary outcomes were defined as: 1) a composite outcome of SAEs including death, cardiac arrest and accidental extubation; and 2) a composite outcome of AEs that directly contribute to morbidity or mortality, including new arrhythmias, hypotension (systolic blood pressure $<100 \mathrm{mmHg}$ or mean arterial pressure $<65 \mathrm{mmHg}$ ), hypertension (systolic blood pressure $>140 \mathrm{mmHg}$ ), desaturation (pulse oximetry $\leq 90 \%$ ), hypoglycaemia (blood glucose $<4 \mathrm{~g} / \mathrm{dl}$ ) and hypothermia (skin temperature $<35.5^{\circ} \mathrm{C}$ ). These composites were selected as they comprise the clinically most significant patient-related AEs associated with IHT.

Five secondary outcomes were defined. The first outcome was miscellaneous complications, defined as biting or blockage of the endotracheal tube, excessive secretions, agitation as measured by the Richmond Agitation Sedation Scale within 30 minutes of arrival in ICU, incomplete muscle relaxation or reversal - defined as lack of full reversal of the muscle relaxant, confirmed with a train of four-ratio of more than $85 \%$, or lack of re-paralysis prior to transfer with half the intubation dose of the muscle relaxant. The second outcome was equipment related AEs, defined as oxygen supply failure, monitor, syringe pump or transport ventilator failure and intravenous line failure (infiltrated or disconnected). The third and fourth outcomes were the total number of AEs and the total number of IHTs complicated by AEs respectively. The fifth

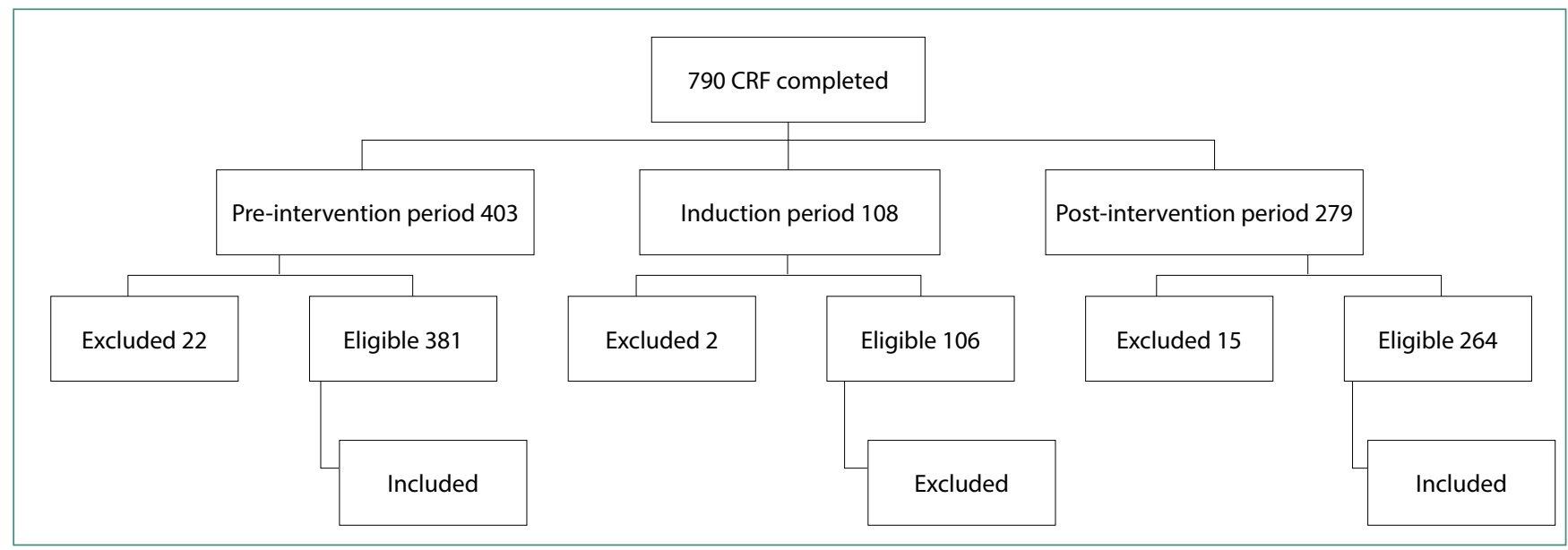

Figure 1: Flow diagram illustrating the breakdown of completed intrahospital transfers included and excluded in the data analysis CRF - case report forms 
outcome was a subjective measure of the percentage of IHTs in need of intervention on and up to 30 minutes after arrival in ICU, as perceived by the receiving ICU practitioner. This outcome was identified to explore the impact of the quality of transfers on the subjectively perceived stability of the patient within and up to the first 30 minutes of arrival in ICU and thus the workload of the receiving ICU practitioner in those same 30 minutes.

\section{Results}

There were 790 case report forms completed over the course of 11 months. There were 381 pre-intervention transfers, 106 induction period transfers, and 264 post-intervention transfers eligible for analysis. Thirty-nine transfers were excluded for being nurse or ICU practitioner led transfers, transfers from the general wards to ICU, or having inadequate data completed on the forms. The induction period IHTs were excluded from the data analysis (Figure 1). The pre- and post-intervention groups were similar regarding patient age, experience level and discipline of lead transporter (predominantly anaesthetist led), reason for ICU admission, timing of IHT and the location from which they were transported. There were more patients intubated and ventilated and more patients on inotropes in the post-intervention group compared to the pre-intervention group (Table I).

There was no difference between the pre- and post-intervention groups for the individual AEs making up the primary outcomes. There was only one SAE during the study period (an accidental extubation during the post-intervention period). There were no deaths, nor non-fatal cardiac arrests (Table II). There were 54 (14.2\%; 95\% confidence interval [Cl] 11-18\%) vs 29 (11\%;

Table I: Demographic characteristics of the included intrahospital transfers

\begin{tabular}{|c|c|c|c|}
\hline & Pre-intervention period & Post-intervention period & Statistical test \\
\hline \multicolumn{4}{|c|}{ Age mean $(95 \% \mathrm{Cl})$} \\
\hline & $38.7(36.9-40.5)$ & $37.4(35.2-39.7)$ & Student's $T=0.8518$ \\
\hline \multicolumn{4}{|c|}{ Level of lead transporter $n(\%)$} \\
\hline Consultant & $51(13.4)$ & $33(12.5)$ & \multirow{5}{*}{$\begin{array}{c}\text { Pearson } \mathrm{Chi}^{2}=2.47 \\
P=0.5\end{array}$} \\
\hline Registrar & $92(24.1)$ & $76(28.8)$ & \\
\hline Medical officer & $223(58.5)$ & $141(53.4)$ & \\
\hline Intern & $12(3.1)$ & $6(2.3)$ & \\
\hline Not reported & $3(0.8)$ & $8(3)$ & \\
\hline \multicolumn{4}{|c|}{ Discipline of lead transporter $n(\%)$} \\
\hline Anaesthesia & $309(81.1)$ & $215(81.4)$ & \multirow{4}{*}{$\begin{array}{c}\text { Pearson } \mathrm{Chi}^{2}=7.638 \\
P=0.4\end{array}$} \\
\hline Emergency medicine & $57(15)$ & $45(17)$ & \\
\hline General surgery & $10(2.6)$ & $2(0.8)$ & \\
\hline Other & $5(1.3)$ & $2(0.76)$ & \\
\hline \multicolumn{4}{|c|}{ Reason for ICU admission $n(\%)$} \\
\hline Postoperative & $313(82.2)$ & $216(81.8)$ & Fisher's exact $=0.917$ \\
\hline Nonoperative & $68(17.8)$ & $48(18.2)$ & \\
\hline \multicolumn{4}{|c|}{ Timing of IHT $n$ (\%) } \\
\hline 08:00 - 15:59 & $166(43.6)$ & $106(40.2)$ & Pearson $\mathrm{Chi}^{2}=0.62$ \\
\hline $16: 00-07: 59$ & $215(56.4)$ & $156(59.1)$ & $P=0.4$ \\
\hline Unknown & $0(0)$ & $2(0.8)$ & \\
\hline \multicolumn{4}{|c|}{ Location being transferred from $n(\%)$} \\
\hline Main theatre & $315(82.7)$ & $213(80.7)$ & $\begin{array}{c}\text { Pearson } \mathrm{Chi}^{2} 0.418 \\
\quad P=0.5\end{array}$ \\
\hline Obstetric theatre & $1(0.3)$ & $4(1.5)$ & $\begin{array}{l}\text { Pearson Chi } 3.181 \\
\qquad P=0.1\end{array}$ \\
\hline Emergency department & $65(17.1)$ & $47(17.8)$ & $\begin{array}{l}\text { Pearson } \mathrm{Chi}^{2} 0.06 \\
\quad P=0.8\end{array}$ \\
\hline \multicolumn{4}{|c|}{ Intubated and ventilated $n(\%)$} \\
\hline Yes & $236(61.9)$ & $190(72)$ & Fisher's exact $=0.008$ \\
\hline No & $142(37.3)$ & $72(27.3)$ & \\
\hline Not reported & $3(0.8)$ & $2(0.8)$ & \\
\hline \multicolumn{4}{|c|}{ Inotropic support $n$ (\%) } \\
\hline Yes & $121(31.8)$ & $102(38.6)$ & Fisher's exact $=0.076$ \\
\hline No & $258(67.7)$ & $160(60.6)$ & \\
\hline Not reported & $2(0.5)$ & $2(0.8)$ & \\
\hline
\end{tabular}

$\mathrm{Cl}$ - confidence interval, $\mathrm{n}$ - number, ICU - intensive care unit, IHT - intrahospital transfer 
Table II: Results of the primary outcomes of serious adverse events and adverse events directly contributing to morbidity and mortality

\begin{tabular}{|c|c|c|c|}
\hline & Pre-intervention & Post-intervention & Statistical test \\
\hline \multicolumn{4}{|c|}{ Serious adverse events ${ }^{\gamma} n$} \\
\hline Yes & 0 & $1 *$ & \multirow[t]{2}{*}{ Test not applied } \\
\hline No & 381 & 263 & \\
\hline \multicolumn{4}{|c|}{ Adverse events directly contributing to morbidity/mortalityt $n(\%)$} \\
\hline Yes & $222(58.3)$ & $148(56.1)$ & \multirow{2}{*}{$\begin{array}{c}\text { Pearson } \mathrm{Chi}^{2}=0.3106 \\
P=0.6\end{array}$} \\
\hline No & $159(41.7)$ & $116(43.9)$ & \\
\hline
\end{tabular}

${ }^{r}$ Death, cardiac arrest, accidental extubation

* One accidental extubation en route

tNew arrhythmias, hypotension (systolic blood pressure $<100 \mathrm{mmHg}$ or mean arterial pressure $<65 \mathrm{mmHg}$ ), hypertension (systolic blood pressure $>140 \mathrm{mmHg}$ ), desaturation (pulse oximetry $\leq$ $90 \%$ ), hypoglycaemia (blood glucose $<4 \mathrm{~g} / \mathrm{dl}$ ) hypothermia (skin temperature $<35.5^{\circ} \mathrm{C}$ )

$\mathrm{n}$ - number

Table III: Results of the secondary outcomes of miscellaneous complications and equipment-related adverse events

\begin{tabular}{|c|c|c|c|}
\hline & Pre-intervention & Post-intervention & Statistical test \\
\hline \multicolumn{4}{|c|}{ Miscellaneous complications ${ }^{\Upsilon} \boldsymbol{n}(\%)$} \\
\hline Yes & $49(12.9)$ & $25(9.5)$ & \multirow{2}{*}{$\begin{array}{c}\text { Pearson } \mathrm{Chi}^{2}=1.7657 \\
P=0.2\end{array}$} \\
\hline No & $332(87.1)$ & $239(90.5)$ & \\
\hline \multicolumn{4}{|c|}{ Equipment-related adverse events* $n(\%)$} \\
\hline Yes & $20(5.2)$ & $5(1.9)$ & \multirow{2}{*}{$\begin{array}{c}\text { Pearson } \mathrm{Chi}^{2}=4.7124 \\
P=0.030\end{array}$} \\
\hline No & $361(94.8)$ & $259(98.1)$ & \\
\hline
\end{tabular}

ҮBiting or blockage of endotracheal tube, excessive secretions, agitation, incomplete muscle relaxation or reversal

*Oxygen supply failure, monitor, syringe pump- or transport ventilator failure, intravenous line failure (infiltrated, disconnected)

$\mathrm{n}$ - number

Cl 7.7-15.4) hypotensive episodes, 115 (30.2\%; Cl 30-35.1\%) vs 88 (33.3\%; Cl 27.9-39.4\%) hypertensive episodes, one (0.26\%; Cl $0-1.8 \%)$ vs two $(0.76 \% ; \mathrm{Cl} 0.1-2.9 \%)$ new arrhythmias, 16 (4.2\%; $\mathrm{Cl} 2.5-6.8 \%)$ vs five $(1.9 \% ; \mathrm{Cl} 0.7-4.4 \%)$ desaturation episodes, 13 (3.4\%; Cl 1.9-5.8\%) vs 12 (4.5\%; Cl 2.6-7.9\%) hypoglycaemic episodes and 86 (22.6\%; Cl 18.8-27.3\%) vs 51 (19.3\%; Cl $15-24.5 \%)$ hypothermic episodes in the pre- vs post-intervention groups. AEs directly contributing to morbidity or mortality as a composite outcome, showed a reduction from 222 transfers (58.3\%; Cl 53.1-63.8\%) pre-intervention, to 148 transfers (56.1\%; Cl 50.2-62.4\%) post-intervention ( $p=0.6$ ) (Table II).

There was mostly no difference between the two groups regarding miscellaneous complications. There were six $(1.6 \%$; $\mathrm{Cl} 0.7-3.4 \%)$ vs five $(1.9 \% ; \mathrm{Cl} 0.7-4.4 \%)$ bitten or blocked endotracheal tubes, seven $(1.8 \% ; \mathrm{Cl} 0.8-3.8 \%)$ vs three $(1.1 \%$; $\mathrm{Cl} 0.3-3.4 \%)$ episodes of excessive secretions and $16(4.2 \% ; \mathrm{Cl}$ $2.6-6.8 \%)$ vs nine $(3.4 \% ; 1.8-6.4 \%)$ agitation episodes in the two groups. There were $19(5 \% ; \mathrm{Cl} 3.4-7.1 \%)$ vs four $(1.5 \% ; \mathrm{Cl}$ $0.6-2.4 \%)$ episodes of incomplete paralysis or reversal in the pre- vs post-intervention group $\left(\mathrm{Chi}^{2}=5.4932, p\right.$-value 0.019). The composite outcome of miscellaneous complications yielded a reduction of 49 (12.9\%; Cl 10.3-14.7\%) pre-intervention to 25 (9.5\%; Cl 8.3-11.1\%) post-intervention ( $p=0.2$ ) (Table III).

Most individual equipment-related AEs did not show a significant difference between the two groups. There were no episodes of oxygen supply failure. There were $10(2.6 \% ; \mathrm{Cl} 1.4-4.8 \%)$ vs two (0.76\%; Cl 0.2-2.9\%) monitor failures, two $(0.52 \%$; Cl 0.1-2.1\%) vs zero syringe pump failures, three $(0.79 \% ; \mathrm{Cl} 0.2-2.4 \%)$ vs two $(0.76 \% ; \mathrm{Cl} 0.2-2.9 \%)$ ventilator failures, and four ( $1 \%$; $\mathrm{Cl} 0.3-2.8 \%)$ vs zero intravenous line failures in the two groups. The composite outcome of equipment related AEs yielded a reduction of 20 complicated transfers $(5.2 \% ; \mathrm{Cl} 3.4-8 \%)$ to five complicated transfers (1.9\%; Cl 0.8-4.5\%; $p=0.03$ ) (Table III).

Each transfer had a possible 21 adverse events. The preintervention group had 400 out of 8001 possible $A E s$ (5\%; CI $3.6-7.4 \%)$. This was reduced to 228 out of 5544 possible AEs in the post-intervention group $(4.1 \%$; $\mathrm{Cl} 2.4-6.8 \% ; p=0.02$ ) (Table

Table IV: Secondary outcomes of overall proportion of adverse events and percentage of transfers associated with adverse events

\begin{tabular}{|c|c|c|c|c|}
\hline \multicolumn{5}{|c|}{ Total number of adverse events: $n$ (\%) } \\
\hline & Adverse events & No adverse events & Total possible adverse events* & Statistical test \\
\hline Pre-intervention & $400(5)$ & $7601(95)$ & $8001(100)$ & \multirow{2}{*}{$\begin{array}{c}\mathrm{Chi}^{2}=5.626 \\
\text { 2-tailed } P=0.02\end{array}$} \\
\hline Post-intervention & $228(4.1)$ & $5316(95.9)$ & $5544(100)$ & \\
\hline \multicolumn{5}{|c|}{ Total number of transfers with one or more adverse events: $n(\%)$} \\
\hline & Adverse events & No adverse events & Total nr of IHT's & Statistical test \\
\hline Pre-intervention & $241(63.3)$ & $140(36.7)$ & $381(100)$ & \multirow{2}{*}{$\begin{array}{c}\text { Pearson } \mathrm{Chi}^{2}=0.465 \\
P=0.5\end{array}$} \\
\hline Post-intervention & $160(60.6)$ & $104(39.4)$ & $264(100)$ & \\
\hline
\end{tabular}

*21 possible adverse events per transfer, $21 \times 381=8001$ in pre-intervention group and $21 \times 264=5544$ in post-intervention group $\mathrm{n}$ - number 
IV). The total number of transfers complicated by one or more $\mathrm{AE}$, showed a reduction from 241 out of 381 transfers $(63.3 \% ; \mathrm{Cl}$ $61.9-65.1 \%)$ to 160 out of 264 transfers $(60.6 \%$; Cl $58.8-63.1 \%$; $p=0.5$ ) (Table IV).

There was a reduction from $132(34.6 \% ; \mathrm{Cl} 31.4-37.8 \%)$ transfers needing intervention within the first 30 minutes of arrival in ICU pre-intervention, to $60(22.7 \% ; \mathrm{Cl} 18-28.2 \%)$ post-intervention (Pearson $\mathrm{Chi}^{2}=11.02 ; p=0.001$ ), as subjectively decided by the receiving ICU practitioner.

\section{Discussion}

Fanara et al. subdivides the IHT-related AE risk factors into human factors (relating to transport teams), equipment factors, organisational factors and patient-related factors. ${ }^{8}$ Patient factors include changes in physiology in response to transport, such as acceleration/deceleration forces, changes in posture, pain and discomfort due to movement, and changes in the environment. The basis of this study is that human, equipment and organisational factors can be mitigated to minimise risk, as patient-related factors are often non-modifiable. However, by including steps to confirm stability and optimisation prior to IHT in both the IHT protocol and checklist, the study's intervention bundle attempted to minimise these patient-related risk factors as much as possible.

Many international guidelines and recommendations focus on the transfer of critically ill patients. ${ }^{16-18}$ These guidelines have the same aim: to improve the quality of transport thereby decreasing AEs. Most of these recommendations focus on the transfer of critically ill patients already admitted to the ICU, being transferred to and from ICU, by ICU trained personnel. Certain authors recommend that a specialist intensivist lead the transport team, ${ }^{19}$ or that transfers should be undertaken by dedicated IHT teams. ${ }^{6}$ In a resource-poor setting the use of a specialist intensivist for this purpose is an inappropriate use of a scarce resource and dedicated IHT teams are not financially viable.

Choi et al. performed a before and after intervention trial, showing a decrease in $\mathrm{AE}$ and SAE from $36.8 \%$ to $22.1 \%$ and from $9.1 \%$ to $5.2 \%$ respectively, after introducing and training ICU nurses to use a transport checklist. ${ }^{20}$ Beigmohammadi et al. showed a difference in AEs during the transport of critically ill patients after using small group clinical course teaching versus large group lecture based training. ${ }^{21}$ Apart from these two studies, very few publications objectively quantify the effects of their proposed guidelines, expert opinions, suggestions and interventions, by showing a decrease in $\mathrm{AE}$ rates.

The core concepts of safe secondary transfer of critically ill patients, includes: the decision to transfer and interdepartmental communication; pre-transfer preparation and stabilisation; considering the mode of transport (for example whether floors need to be crossed); adequately trained transport personnel; correct equipment, drugs, monitoring, handover and documentation. ${ }^{22}$ This study's bundle of interventions attempted to address all of these aspects, aiming to minimise the human, equipment and organisational risk factors contributing to AEs. Structured handover is paramount, as recent local research has shown that implementing a "standardised handoff protocol" leads to fewer interruptions, improved attendance and improved information-sharing during handover to the ICU team. ${ }^{23}$

The reduction in episodes of incomplete reversal or paralysis (5\%; Cl 3.4-7.1\% vs $1.5 \%$; $\mathrm{Cl} 0.6-2.4 \% ; p=0.019)$ is an example of a minimised human-related risk factor. The intervention bundle managed to reduce equipment-related AEs from $5.2 \%(\mathrm{Cl}$ $3.4-8 \%)$ to $1.9 \%(\mathrm{Cl} 0.8-4.5 \%)(p=0.03)$. This is an important finding, as in multiple studies, equipment-related $A E s$ accounted for up to one third to half of AEs. ${ }^{5,9,24}$ Overall, the intervention led to a decrease in the total number of $\mathrm{AEs}(5 \%$; $\mathrm{Cl} 3.6-7.4 \%$ vs $4.1 \%$; Cl 2.4-6.8\%; $p=0.02$ ).

This study was not adequately powered to detect a reduction in the rate of individual AEs. The study showed an absolute reduction, across all measured composite variables. However, it is not clear why the reduction in the overall number of IHTs complicated by AEs did not reach statistical significance $(63.3 \%$; Cl $61.9-65.1 \%$ vs $60.6 \% ; \mathrm{Cl} 58.8-63.1 \% ; p=0.5)$, despite the significant reduction in the overall number of AEs. Firstly the sample size was potentially too small, despite doing a power calculation prior to data collection. A second possibility is the patient-related risk factors for AEs that Fanara et al. described. ${ }^{8}$ These physiological changes in response to an IHT, leading to AEs, are likely non-modifiable and may play a larger role in AEs than the human, equipment and organisational factors, that were addressed with this intervention bundle. Thirdly, the transfers in the post-intervention group were done in much sicker patients, as there was a higher proportion of patients intubated and ventilated and a higher proportion of patients on inotropic support in the post-intervention group. It has been demonstrated that sicker patients experience more AEs. Patients with APACHE II scores of 20 or more vs patients with APACHE II scores of 11 or less was identified as a patient-related risk factor for the development of AEs. ${ }^{25}$ Unfortunately, severity scores such as the APACHE II or SOFA scores, were not captured during data collection. A study performed in Johns Hopkins hospital, demonstrated that only $17 \%$ of AEs happened during level three transfers (i.e. high care patients and patients requiring minimal ventilatory support), whereas $83 \%$ of AEs occurred during level four transfers (i.e. haemodynamically unstable patients and those requiring significant ventilatory support). ${ }^{6}$ ParmentierDecrucq's univariate analysis showed that the need for PEEP of $>6 \mathrm{cmH}_{2} \mathrm{O}$ and need for treatment modification prior to transfer, were associated with a higher incidence of AEs. ${ }^{9}$ Fourthly, a significant proportion of patients included in both groups were not intubated and ventilated and even fewer patients were on inotropic support. It could be postulated that these unintubated, non-ventilated, haemodynamically stable patients were too well and thus had fewer AEs, regardless of whether they were included in the pre- or post-intervention groups. The study was not adequately powered to do sub-analyses of patients on 
invasive ventilation or inotropic support to explore this further. A fifth reason may be that IHTs in both groups were predominantly done from the main operating theatre to ICU $(82.7 \%$ preintervention vs $80.7 \%$ post-intervention). The distance between the main operating theatre and ICU in both hospitals is a very short distance and in both instances, does not include crossing floors via an elevator. It is possible that longer distance IHTs, including crossing floors, may have yielded a larger difference between the two groups. Finally, most of the IHTs in this study had lead transporters from the anaesthesia department $(81.1 \%$ preintervention vs $81.4 \%$ post-intervention). A previous publication demonstrated a lower incidence of equipment-related AEs if IHTs were executed by anaesthesia providers. ${ }^{9}$ The results from our study may have been more significant if there were more IHTs done with members of other departments as lead transporters.

It was interesting to note that there was a reduction in perceived necessity for interventions in the first 30 minutes after arrival in ICU in the two groups, from $34.6 \%$ (Cl $31.4-37.8 \%$ ) to $22.7 \%$ (Cl 18-28.2\%), as observed by the receiving practitioner. This implies that even if an IHT was not associated with an objectively quantified $A E$, the receiving practitioner subjectively regarded the patients as more stable in the post-intervention group, despite this group having sicker patients. This is, however, a subjective, non-blinded measure and should be interpreted with caution.

This study has many limitations. The IHT protocol did not include continuous end-tidal capnography, included in many international IHT recommendations. 8,10,14,15 The authors could only secure continuous end-tidal capnography for one of the two study centres, so it was not included in the transport protocol. It should be included in IHT protocols and used whenever available. Each centre should develop its own protocols, dependent on available resources. The IHT protocol does not include standard laryngoscopes, endotracheal tubes and stylets. In an ideal setting, where all IHTs are performed by senior staff with the skill to intubate under suboptimal conditions and no resource limitations, these should be included in transfer equipment, again emphasising the need for centres to individualise IHT protocols.

Due to the nature of the before-after trial design used, there are multiple sources of possible bias. Participants and transporters were not blinded to the intervention, as the intervention was based on using the newly developed protocol and checklist to transport participants. This may contribute to error from the Hawthorne effect and observer bias. ${ }^{26}$ Due to the convenience sampling method of consecutive transfers into units and the lack of a control group, it was not possible to match pre- and post-intervention groups exactly, neither demographically, nor based on primary pathology or severity of disease. This led to the patients in the post-intervention group being sicker, which may have led to selection bias. ${ }^{26}$ This study did not detect changes in incidence of AEs that occurred beyond the first 30 minutes after arrival in ICU as data collection stopped after 30 minutes of arrival in ICU. These long-term complications include deep vein thrombosis, atelectasis, postoperative pulmonary complications, ventilator-associated pneumonia and increased length of ICU stay. This requires investigation in future studies, with more prolonged follow up. This study was conducted in a single metropole and would therefore require external validation.

\section{Conclusion}

The incidence of adverse events during IHT remains alarmingly high and cannot be ignored. This study failed to demonstrate an overall decrease in IHTs complicated by AEs. However, the bundle of interventions did facilitate a decrease in the overall number of $A E s$, equipment-related $A E s$ and the need for intervention within the first 30 minutes of arrival in ICU post IHT. A bundle of interventions should be considered for use by hospitals who regularly embark on IHT of the critically ill, especially if the institution does not have a formalised IHT protocol. This study focused on IHT, performed by medical doctors, with a special focus on IHT between the operating theatre and ICU. The universality of the risk factors, hazards and AEs associated with the transfer of all critically ill patients, be it intra- or interhospital, as well as the mitigating factors and safety precautions that should be put in place, cannot be overemphasised. Taking the study's results into account, the authors suggest that the methods of improving safety during IHT demonstrated during this study should be applied across all disciplines and skill sets involved in transfers of critically ill patients.

\section{Acknowledgements}

We acknowledge the contributions of GS Henderson for his input in creating the transport video and RP von Rahden for his input in creating the transport protocol and checklist.

\section{Conflict of interest}

All authors have no conflicts of interests to declare.

\section{Funding source}

None.

\section{Ethic approval}

Ethical approval was obtained from both hospitals' management, the University of KwaZulu-Natal's Biomedical Research Ethics Committee (BREC reference number BFC 047/17), and the KwaZulu-Natal Department of Health.

\section{ORCID}

L Geldenhuys (ID) https://orcid.org/0000-0002-7695-2572

R Wise (D) https://orcid.org/0000-0001-5237-5582

R Rodseth (iD https://orcid.org/0000-0002-3779-7805

\section{References}

1. Gray A, Bush S, Whiteley S. Secondary transport of the critically ill and injured adult. Emergency Medicine Journal. 2004;21(3):281-5. https://doi.org/10.1136/ emj.2003.005975.

2. Dunn $M$, Gwinnutt $C$, Gray A. Critical care in the emergency department: patient transfer. Emergency Medicine Journal. 2007;24(1):40-4. http://dx.doi. org/10.1136/emj.2006.042044.

3. Schwebel C, Clec'h C, Magne S, et al. Safety of intrahospital transport in ventilated critically ill patients: a multicenter cohort study. Critical Care Medicine. 2013;41(8):1919-28. https://doi.org/10.1097/CCM.0b013e31828a3bbd.

4. Bercault N, Wolf M, Runge I, Fleury J-C, Boulain T. Intrahospital transport of critically ill ventilated patients: a risk factor for ventilatorassociated pneumonia-a matched cohort study. Critical Care Medicine. 2005;33(11): 2471-8. https://doi.org/10.1097/01.CCM.0000185644.54646.65. 
5. Papson JP, Russell KL, Taylor DM. Unexpected events during the intrahospital transport of critically ill patients. Academic Emergency Medicine. 2007;14(6):574-7. https://doi.org/10.1197/j.aem.2007.02.034.

6. Kue R, Brown P, Ness C, Scheulen J. Adverse clinical events during intrahospital transport by a specialized team: a preliminary report. American Journal of Critical Care. 2011;20(2):153-62. https://doi.org/10.4037/ajcc2011478.

7. Gillman L, Leslie G, Williams $T$, et al. Adverse events experienced while transferring the critically ill patient from the emergency department to the intensive care unit. Emergency Medicine Journal. 2006;23(11):858-61. http:// dx.doi.org/10.1136/emj.2006.037697.

8. Fanara B, Manzon C, Barbot O, Desmettre T, Capellier G. Recommendations for the intra-hospital transport of critically ill patients. Critical Care. 2010;14(3):R87. https://doi.org/10.1186/cc9018.

9. Parmentier-Decrucq E, Poissy J, Favory R, et al. Adverse events during intrahospital transport of critically ill patients: incidence and risk factors. Annals of Intensive Care. 2013;3(1):10. https://doi.org/10.1186/2110-5820-3-10.

10. Brunsveld-Reinders AH, Arbous MS, Kuiper SG, De Jonge E. A comprehensive method to develop a checklist to increase safety of intra-hospital transport of critically ill patients. Critical Care. 2015;19(1):1-10. https://doi.org/10.1186/ s13054-015-0938-1.

11. Hales B, Terblanche M, Fowler R, Sibbald W. Development of medical checklists for improved quality of patient care. Int J Qual Health Care. 2008;20(1):22-30. https://doi.org/10.1093/intqhc/mzm062.

12. Warren J, Fromm Jr RE, Orr RA, Rotello LC, Horst HM. Guidelines for the inter-and intrahospital transport of critically ill patients. Critical Care Medicine. 2004;32(1):256-62. https://doi.org/10.1097/01.CCM.0000104917.39204.0A

13. Quenot JP, Milési C, Cravoisy A, et al. Intrahospital transport of critically ill patients (excluding newborns) recommendations of the Société de Réanimation de Langue Française (SRLF), the Société Française d'Anesthésie et de Réanimation (SFAR), and the Société Française de Médecine d'Urgence (SFMU). Annals of Intensive Care. 2012;2. https://doi.org/10.1186/2110-5820-2-1.

14. Jarden RJ, Quirke S. Improving safety and documentation in intrahospital transport: development of an intrahospital transport tool for critically ill patients. Intensive and Critical Care Nursing. 2010;26(2):101-7. https://doi. org/10.1016/j.iccn.2009.12.007.

15. Comeau OY, Armendariz-Batiste J, Woodby SA. Safety First! Using a Checklist for Intrafacility Transport of Adult Intensive Care Patients. Crit Care Nurse. 2015;35(5):16-25. https://doi.org/10.4037/ccn2015991.
16. Australasian College for Emergency Medicine (ACEM), Australian and New Zealand College of Anaesthetists (ANZCA), College of Intensive Care Medicine of Australia and New Zealand (CICM). Guidelines for Transport of Critically III Patients. 2013. http://www.anzca.edu.au/resources/professional-documents/ pdfs/ps52-2013-guidelines-for-transport-of-critically-ill-patients.pdf/at_ download/file.

17. Whiteley S, Macartney I, Mark J, Barratt H, Binks R. Guidelines for the transport of the critically ill adult, 3rd edition, The Intensive Care Society 2011.

18. Knight PH, Maheshwari N, Hussain J, et al. Complications during intrahospital transport of critically ill patients: Focus on risk identification and prevention. International Journal of Critical IIIness and Injury Science. 2015;5(4):256-64. https://doi.org/10.4103/2229-5151.170840.

19. Venkategowda PM, Rao SM, Mutkule DP, Taggu AN. Unexpected events occurring during the intra-hospital transport of critically ill ICU patients. Indian Journal of Critical Care Medicine. 2014;18(6):354-7. https://doi. org/10.4103/0972-5229.133880.

20. Choi HK, Do Shin S, Ro YS, et al. A before- and after-intervention trial for reducing unexpected events during the intrahospital transport of emergency patients. The American Journal of Emergency Medicine. 2012;30(8):1433-40. https://doi.org/10.1016/j.ajem.2011.10.027.

21. Beigmohammadi MT, Rahimi M, Nabavian O, et al. Clinical course teaching in transport of critically ill patients: Small group methods. Acta Medica Iranica. 2016;54(9).

22. Kulshrestha A, Singh J. Inter-hospital and intra-hospital patient transfer: Recent concepts. Indian Journal of Anaesthesia. 2016;60(7):451-457. https://doi. org/10.4103/0019-5049.186012.

23. Van der Walt J, Scholl A, Joubert I, Petrovic MA. Implementation of a postoperative handoff protocol. Southern African Journal of Anaesthesia and Analgesia. 2016;22(6):190-4. https://doi.org/10.1080/22201181.2016.1244317.

24. Waydhas C. Intrahospital transport of critically ill patients. Critical Care. 1999;3(5):R83-9. https://doi.org/10.1186/cc362.

25. Jia L, Wang H, Gao Y, Liu H, Yu K. High incidence of adverse events during intra-hospital transport of critically ill patients and new related risk factors: a prospective, multicenter study in China. Critical Care. 2016;20:12. https://doi. org/10.1186/s13054-016-1183-y.

26. Ho AM, Phelan R, Mizubuti GB, et al. Bias in before-after studies: narrative overview for anesthesiologists. Anesthesia and Analgesia. 2018;126(5):1755-62. https://doi.org/10.1213/ANE.0000000000002705.

\section{Appendix 1}

\section{PMB Protocol for the intrahospital transport of critically ill patients:}

The critically ill patient being transferred from theatre or the emergency department to ICU needs constant, invasive organ monitoring and support. Critically ill patients that are defined as being:

- haemodynamically unstable,

- on inotropes,

- intubated and ventilated (for ventilatory, metabolic, neurological or airway support).

Such patients have to be accompanied by at least one medical doctor capable of providing emergency organ support during transfer.

This includes being able to:

- ventilate via a bag-valve resuscitator and transport ventilator,

- use airway adjuncts \& alternatives,

- manage inotrope-infusions and boluses, and

- administer advanced life support.

A minimum of two (qualified) people has to accompany the patient, as per international guidelines.

\section{Monitoring:}

A transport monitor has to be connected to every critically ill patient. The battery has to be at least $50 \%$ charged.

Adequate monitoring for every critically ill patient includes:

- $\mathrm{SpO}_{2}$,

- ECG,

- NIBP cycling at least every 2.5 minutes, and

- invasive BP reading if on inotropic infusions.

\section{Equipment:}

- An oxygen cylinder, size'E' with a functional gauge, compatible with transport ventilator and oxygen tubing, at least $50 \%$ full, (i.e. $75 \mathrm{Bar} / 1100 \mathrm{psi}$ ). If the patient is to be transported via an elevator or ambulance, a second cylinder is necessary (any size). If no functional gauge is available, open a new, sealed size 'E' oxygen cylinder. Confirm that there is a suitable connection for both oxygen tubing and the ventilator's oxygen pipeline on the cylinder.

- A bag-valve resuscitator with oxygen tubing must accompany all patients, even if transport ventilator is in use or the patient is not intubated.

- Transport ventilator with correct circuit, battery fully charged. 


\begin{tabular}{ll}
\hline Equipment for accidental extubation: \\
\hline Not a difficult airway: & Difficult/threatened airway: \\
\hline - Oropharyngeal airway & - Pre packed cricothyroidotomy set \\
- Face mask & Or \\
- Laryngeal mask airway & - Size 11 blade \& scalpel handle \\
& And \\
& - Size 6 cuffed ETT \\
& - 'Puffer' syringe \\
\hline
\end{tabular}

\section{Patient preparation:}

- Head-to-toe examination to confirm stability and readiness for transfer.

- Document vital signs pre-transfer.

- Place an oropharyngeal airway for every intubated patient directly prior to transfer and remove on arrival in ICU.

- Confirm correct ETT placement (via auscultation) and depth prior to transfer.

- Confirm placement of

- urinary catheter

- nasogastric tube (if Intubated and ventilated), and

- IV-line(s) (see below).

- Confirm adequate, synchronised ventilation on the transport ventilator.

\section{Drugs:}

- At least 1 large bore IV-line, able to run freely.

- In the case of any infusions running during transfer, a second IV-line has to be in situ for giving boluses of other drugs.

- At least $200 \mathrm{ml}$ of crystalloid has to be connected to the "bolus" line to flush drugs in.

\section{Or}

- $20 \mathrm{ml}$ "flush" syringe with normal saline for the transfer.

- Inotropic infusions must be running via a central venous line.

- Inotropic infusions must be controlled via infusion pumps or syringe drivers.

- If a patient is on inotropic support, an arterial line must be in situ, unless the equipment or skill is unavailable - inform the ICU consultant receiving the patient prior to transfer.

- If unable to place CVP due to lack of skill/equipment, and needing inotropic support: Adrenaline $20 \mathrm{mcg} / \mathrm{ml}$ (not more concentrated than this) via an infusion pump or syringe driver on a dedicated IV-line. Inform ICU consultant receiving the patient prior to transfer.

- Adrenaline infusion: if at any point during the procedure the adrenaline infusion was $0.2 \mathrm{mcg} / \mathrm{kg} / \mathrm{min}$ or more, or the infusion was stopped less than 30 minutes prior to transfer, the adrenaline infusion has to be running for the transfer (even if the patient is weaned off adrenaline prior to transfer), as patients become unstable on transfer due to acute physiological changes during movement.

\begin{tabular}{ll}
\hline Muscle relaxants: & \\
\hline $\begin{array}{l}\text { Airway protection or respiratory } \\
\text { compromise/ paralyses superior } \\
\text { to reversal*: }\end{array}$ & $\begin{array}{l}\text { No airway or respiratory } \\
\text { compromise: }\end{array}$ \\
\hline - Reparalyse: give $1 / 2$ the intubation & - Reversal: \\
dose of NDMR used, directly & - Neostigmine $0.04 \mathrm{mg} / \mathrm{kg} \&$ \\
before transfer & Glycopyrrolate $0.01 \mathrm{mg} / \mathrm{kg}$ \\
- Inform receiving ICU doctor re & Confirm with Train-of-Four \\
paralyses & ration $>85 \%$ on nerve \\
& stimulator \\
& - Inform receiving ICU doctor \\
& re reversal \\
\hline
\end{tabular}

*Situations where paralysis may be deemed superior to reversal, include patients needing lowering of intracranial pressures (ICP), such as patients for neuroprotection post head injury. The critically ill eclamptic patient that is intubated and ventilated often needs both airwayand neuroprotection.

The following drugs has to be given to all intubated patients 5-10 minutes prior to transfer, additional to analgesia already given, unless specifically requested not to give by consultant in charge of the patient, or alternative requested by consultant:

Opioid based analgesia:

- Fentanyl $2 \mathrm{mcg} / \mathrm{kg}$ prior to transfer and further $1 \mathrm{mcg} / \mathrm{kg}$ in a vial or syringe available during transfer.

Sedation:

- Ketamine $0.5 \mathrm{mg} / \mathrm{kg}$

- Midazolam $1 \mathrm{mg}$ bolus followed by $1 \mathrm{mg}$ bolus 5 minutes later. If more sedation needed, give $1 \mathrm{mg}$ increments up to a maximum of $5 \mathrm{mg}$ Midazolam - monitor closely for haemodynamic effects of Benzodiazepine and opiate coadministration, may need to increase inotrope infusion and stop any further Midazolam boluses.

- Volatiles must be weaned to $<0.3$ MAC multiple before decision made that patient is adequately sedated with IVdrugs for transfer.

Emergency drugs drawn up:

- Adrenaline $1 \mathrm{mg}$ diluted in $9 \mathrm{ml} 0.9 \%$ saline, to $10 \mathrm{ml}$ $(100 \mathrm{mcg} / \mathrm{ml})$

Backup induction drugs:

- Induction agent: ampule of Etomidate (dose $0.2 \mathrm{mg} / \mathrm{kg}$ ) with syringe and needle.

- Muscle relaxant: ampule of Suxamethonium (dose $1 \mathrm{mg} / \mathrm{kg}$ ) with syringe and needle. If Suxamethonium is contraindicated, 2 ampules of Rocuronium (dose $1 \mathrm{mg} / \mathrm{kg}$ ) with syringe and needle.

\section{Communication:}

- Telephonically or personally inform ICU staff of:

- imminent arrival in ICU,

- condition of patient,

- infusions patient is receiving, including inotropic infusion concentrations, and 
- send somebody ahead to clear a path to ICU (especially if floors need to be crossed, to hold the elevator).

\section{On arrival in ICU:}

- Confirm stability of patient post transfer.

- Document post transfer vital signs.

- Verbally hand patient over to attending ICU doctor.

- Confirm adequate, synchronised ventilation on ICU ventilator.

- Communicate and document any adverse events during transfer.

- Confirm that transport monitor and ventilator is plugged in for battery charging.

- Confirm that oxygen cylinder is closed and still adequate for next transfer.

\section{Special considerations:}

- Cervical spine unstable / not cleared:
- Formal 5 person log roll as per ATLS principles every time patient is moved.

- Stabilise c-spine for transport: hard collar / Philladelphia collar / sandbags.

- Manual in line stabilisation when airway manipulated

- Neuroprotection for patients with (suspected) head injuries / eclampsia / any other cause of cerebral oedema or raised intracranial pressure:

- 30 degrees head up position.

- No circumferential ties around neck.

- Confirm ETCO2 4.7-5.3 kPa prior to transfer.

- $\mathrm{MAP}>80 \mathrm{mmHg}$.

- Normoglycaemia.

- Muscle paralysis to avoid coughing on ETT or fighting the ventilator.

\section{Appendix 2}

Checklist for the intrahospital transport of critically ill patients to ICU:

\begin{tabular}{|l|l|l|}
\hline \multicolumn{2}{|l|}{ Demographic Data: } \\
\hline Name: & Tospital number: \\
\hline Date: & Primary location: \\
\hline Lead transporter level: Intern/MO/Reg/Consult & Number of personnel for transfer: \\
\hline
\end{tabular}

\begin{tabular}{|l|l|l|l|l|l|}
\hline \multicolumn{5}{|c|}{ Equipment: } & ECG \\
\hline Transport monitor & & $\mathrm{SpO}_{2}$ probe & Transport box \\
\hline NIBP cycling $2.5 \mathrm{~min}$ & & IBP (on inotropes) & & \\
\hline Syringe driver & & Airway equipment as per IHT protocol & \\
\hline 'E' $\mathrm{O}_{2}$ cylinder \& functional gauge $(>75 \mathrm{Bar})$ & & Transport ventilator \& circuit & \\
\hline Bag-valve resuscitator with $\mathrm{O}_{2}$ tubing & & Batteries fully charged & \\
\hline
\end{tabular}

\begin{tabular}{|c|c|c|c|c|c|}
\hline \multicolumn{6}{|c|}{ Patient Preparation: } \\
\hline OPA inserted & & Confirm ETT placement & & ETT depth @ teeth & $\mathrm{cm}$ \\
\hline Urinary catheter & & NG-tube if indicated & & IV-lines functional & \\
\hline A-line (if indicated) & & CVP (if indicated) & & Tidal volume: & $\mathrm{ml}$ \\
\hline $\mathrm{SpO}_{2}$ & $\%$ & Heart rate (bpm) & & $\mathrm{BP}(\mathrm{mmHg})$ & I \\
\hline Glucose (mmol/L) & & Temperature & ${ }^{\circ} \mathrm{C}$ & $\mathrm{MAP}(\mathrm{mmHg})$ & \\
\hline Adrenaline: & $\mathrm{mcg} / \mathrm{kg} / \mathrm{min}$ & $\mathrm{FiO}_{2}$ & $\%$ & Patient stable & \\
\hline
\end{tabular}

Transport Drugs:

\begin{tabular}{|c|c|c|}
\hline Crystalloid flush & Etomidate ampule & Sux ampule \\
\hline \multicolumn{3}{|c|}{ Rocuronium ampules x2 (if Sux contra-indicated) } \\
\hline \multicolumn{3}{|c|}{ Adrenaline infusion running if : $\geq 0.2 \mathrm{mcg} / \mathrm{kg} / \mathrm{min}$ at any time / stopped $<30 \mathrm{~min}$ prior to transfer } \\
\hline Emergency drugs: & \multicolumn{2}{|c|}{ Adrenaline $1 \mathrm{mg}$ in $9 \mathrm{ml} 0.9 \%$ saline in $10 \mathrm{ml}$ syringe $=100 \mathrm{mcg} / \mathrm{ml}$} \\
\hline \multirow{2}{*}{$\begin{array}{l}\text { Sedation given to all } \\
\text { intubated patients: }\end{array}$} & Ketamine $0.5 \mathrm{mg} / \mathrm{kg}$ & Fentanyl $2 \mathrm{mcg} / \mathrm{kg}$ \\
\hline & Midazolam $1 \mathrm{mg}$ increments & ET AA $<0.3$ MAC \\
\hline \multirow{2}{*}{$\begin{array}{l}\text { Muscle paralysis for all } \\
\text { patients: }\end{array}$} & \multicolumn{2}{|c|}{ Reparalyse: $50 \%$ NDMR intubation dose 5 min before transfer or } \\
\hline & \multicolumn{2}{|c|}{ Reversal with Neostigmine $0.04 \mathrm{mg} / \mathrm{kg}$ \& Glycopyrrolate $0.01 \mathrm{mg} / \mathrm{kg}$} \\
\hline
\end{tabular}




\begin{tabular}{|c|c|c|c|c|c|}
\hline \multicolumn{6}{|c|}{ Communication: } \\
\hline \multicolumn{5}{|c|}{ Inform ICU staff of ETA, condition of patient, needs incl sedatory \& inotropic infusions } & \\
\hline \multicolumn{5}{|c|}{ Send somebody to clear path to ICU, especially to hold elevator } & \\
\hline \multicolumn{6}{|c|}{ Arrival in ICU: } \\
\hline $\mathrm{SpO}_{2}$ & $\%$ & \multicolumn{2}{|l|}{ Heart rate (bpm) } & $\mathrm{BP}(\mathrm{mmHg})$ & / \\
\hline \multicolumn{4}{|c|}{ Handover to ICU doctor \& nursing staff } & Equipment charging & \\
\hline \multicolumn{3}{|c|}{ Communicate \& document adverse events } & & $\mathrm{O}_{2}$ cylinder closed & \\
\hline \multicolumn{6}{|c|}{ Special Considerations (refer to IHT protocol): } \\
\hline \multicolumn{3}{|c|}{ C-spine unstable / not cleared } & \multicolumn{2}{|c|}{ Neuroprotection } & \\
\hline
\end{tabular}

\section{Appendix 3}

\section{Inventory of intrahospital transport box}

\section{Airway \& ventilation equipment}

Bag-valve resuscitator

Oxygen tubing

Oropharyngeal airways

Face masks

Laryngeal mask airways, IGel

Cricothyroidotomy set:

Size 11 blade

ETT, size 6, cuffed

'Puffer' syringe

Adhesive tape

Trache tape

\section{Drug administration equipment}

Intravenous cannulae

Alcohol swabs

Adhesive dressing

Short line

$0.9 \%$ saline flush

$2 \mathrm{ml}$ syringes

$5 \mathrm{ml}$ syringes

$10 \mathrm{ml}$ syringes

Needles (yellow or green)

\section{Transport drug container}

Etomidate

Suxamethonium

Rocuronium

Adrenaline

Fentanyl $\mathrm{x} 1$

$\mathrm{x} 1$

x3 (sizes 2-4)

x3 (size 3-5)

x3 (size 3-5)

$\mathrm{x} 1$

$\mathrm{x} 1$

$\mathrm{x} 1$

$\mathrm{x} 1$ roll

$\mathrm{x} 1$ roll

x6 (x2 16G, x2 18G, x2 20G)

$x 4$

$x 1$

$\mathrm{x} 1$

$\mathrm{x} 4$ plastic ampules

$\mathrm{x} 2$

$x 2$

$x 4$

$x 5$

x1 ampule

$\mathrm{x} 1$ ampule

$\mathrm{x} 2$ ampules

$\mathrm{x} 1$ ampule

$\mathrm{x} 1$ ampule (2 ml) 